\title{
Time-domain just-before-test verification method to detect failures and ensure the measurement accuracy for conducted emissions and immunity tests
}

\author{
Marc Pous \\ Universitat Politècnica de Catalunya \\ Barcelona, Spain \\ email: marc.pous@upc.edu
}

\author{
Marco Azpúrua \\ Universitat Politècnica de Catalunya \\ Barcelona, Spain \\ email: marco.azpurua@upc.edu
}

\author{
Ferran Silva \\ Universitat Politècnica de Catalunya \\ Barcelona, Spain \\ email: ferran.silva@upc.edu
}

\author{
Soydan Çakir \\ TUBITAK UME \\ Gebze, Turkey \\ soydan.cakir@tubitak.gov.tr
}

\author{
Osman Şen \\ TUBITAK UME \\ Gebze, Turkey \\ osman.sen@tubitak.gov.tr
}

\begin{abstract}
It is mandatory for EMC test laboratories to apply verification methodologies to ensure that they are performing the tests properly. However, the time-consumption of most of the procedures or its low accuracy causes low-impact and consequently a poor benefit for laboratories. With the aim to improve the performance of the current verification methods, a just-before-test methodology based on time-domain measurements is proposed in this publication. In order to improve the accuracy and detect failures for conducted emissions and immunity tests, arbitrary waveforms and full time-domain EMI measurement methods are combined. Hence, it is proposed to use multi-tone and pulsed waveforms to evaluate the performance of the conducted emissions test, including the receiver with normative detectors, paths and devices like LISN. On the other hand, for the immunity test, a time-domain EMI system based on the use of an oscilloscope is employed to identify defects on the amplifier, RF generator, devices like CDNs or paths. The methodologies are explained and validated through different simulated failure scenarios. Where we can identify a proper performance of the test bench or defects like poor grounding or coupling devices malfunctions.
\end{abstract}

Keywords-time-domain measurements, electromagnetic interference, conducted emissions, conducted immunity, verification

\section{INTRODUCTION}

In Electromagnetic Compatibility (EMC) testing, the confidence on the results is based on the uncertainty calculations and the certainty that the test setup and the instrumentation is performing properly. In order to check that the test bench is ready for testing a verification procedure is recommended before the start of each test. According to ISO/IEC 17025 [1], in addition to other actions like laboratory inter-comparison, test laboratories should employ these verification methods [2] and [3], however, usually, the methods are slow and evaluate partially or with a high uncertainty the performance of the test bench.

The research work presented in this paper, which is part of the European research project RFmicrowave [4], aims for developing novel just-before-test verification methodologies. In fact, the main objective is to create novel just-before-test methods capable of identifying common failures that recurrently occurs during EMC tests, focusing on conducted emissions and immunity testing. Therefore, we propose fast methods that delay shortly the day-work at EMC test laboratories but at the same time are feasible to identify setup errors or defects on the used instrumentation [5] and [6]. Currently, many times the testing workbench is verified in different stages including several measurement and instrumentation. For instance, paths are evaluated employing instrumentation like vector network analyzers (VNA), which are adding extra paths to the earth disabling us to identify grounding problematics. Moreover, VNA is expensive instrumentation with sensitive input stages and usually are used without using high RF power at the verification stage to avoid instrumentation damage, meaning that the verification is done partially.

Otherwise, the receivers are evaluated directly connecting radio frequency generators and most of the times using continuous wave $(\mathrm{CW})$ signals to verify the receivers although we know that it is essential to check the normative detectors such as the quasi-peak or the CISPR average. The problem of using CW waveforms as input signals is that they do not have a different response when the weighing detectors are employed, so usually, the normative detector is not verified. In this paper, with the aim to overcome these limitations for the evaluation of the conducted emissions, novel approaches based on time-domain measurements and the use of arbitrary waveform generator (AWG) hve been considered. Employing multi-toned waveforms and pulses to evaluate the instrumentation, Line Impedance Stabilisation Network (LISN), cables, loads and grounding conditions.

Nevertheless, if we get to focus on considering immunity testing like IEC 61000-4-6 [7], a method to rapidly evaluate all the test is necessary. Including the evaluation of the full chain; the generator, amplifier, power meter, directional coupler, paths and Coupling and Decoupling Networks (CDN). Obviously, this is not an easy task and many laboratories employ different measurement to verify this test, resulting in long procedures. Alternatively, test laboratories monitor the forward power, however, full verification of the test bench is incomplete and failures during the test can still occur. To overcome these deficiencies, in the work we propose a novel verification method based on time domain measurements, which allow us to use low-cost instrumentation as oscilloscopes to evaluate quickly the full chain of the conducted immunity test. 


\section{METHODOLOGY}

The methodology applied have some shared development between the conducted emissions and immunity test, however, it has been split into two different subsections to relate the procedure with the results section.

\section{A. Conducted emissions}

As it has been stated at the introduction section, the goal of the just-before-test measurements is to be capable of evaluating the accuracy of the receiver according to the normative detectors, and at the same time to identify possible defects at the test bench. Including grounding failures or apparatus damage or erroneous correction factors. For this purpose, a method based on the use of AWG configuring multi-tone signal or a representative EUT interference waveform will be used as the reference source in combination with the time-domain receiver. Although other types of instrumentation like frequency sweep can be used to perform the just-before-test evaluation, using time-domain instrumentation like oscilloscopes will improve the speed and capabilities of the methodology. Hence, the AWG is replacing the EUT and connected to the LISN, while the RF output of the LISN is connected to the time-domain EMI receiver, as it can be seen in the schematic provided in Fig. 1.

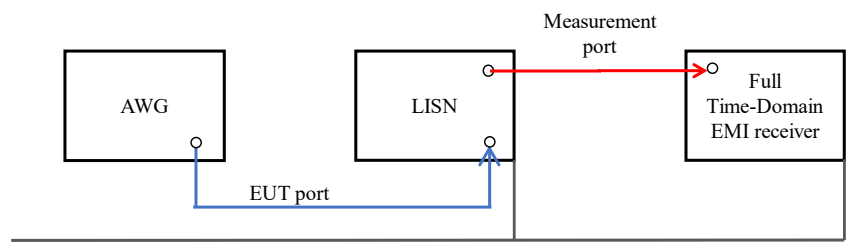

Reference ground plane

Fig. 1. Schematic of the just-before-test verification method for conducted emissions

1) Arbitrary waveform generator (AWG) reference source

The reason to employ an AWG is that we can set the source according to our verification purpose, speeding up the procedure and at the same time focusing on the amplitude accuracy close to a desired limit line level [8]. The waveforms used in the results section are a multi-tone and a representative EUT interference. These signals will allow us to evaluate the full frequency band defined at CISPR standards with a single excitation. For instance, in this paper, the work is focused on conducted emissions frequency band defined as Band B according to CISPR 16-1-1 standard.

\section{a) Multi-tone signal}

The first proposed waveform is a synthesized multi-tone signal as the excitation. The periodic signal $x(t)$ is formed by superposition tones with arbitrary amplitude, frequencies and phases according to the following equation,

$$
x(t)=\sum_{i=1}^{N_{\text {tones }}} A_{i}\left(f_{i}\right) \sin \left(\left(2 \pi f_{i}\right) t+\phi_{i}\left(f_{i}\right)\right)
$$

where $A_{i}, f_{i}$ and $\phi_{i}$ are, the amplitude, frequency, and phase of the $i$-th tone and $N_{\text {tones }}$ is the number of tones generating the signal $x(t)$. The independent control over the amplitude and phase of each tone enables us to control the crest factor in the time-domain [9]. The signal is sampled obtaining a time discrete signal $x[n]$, where $\mathrm{n}=0,1,2 \ldots$ is the integer variable used as the time step index. In Fig. 2, we can find the computed spectrum from the calculated multi-tone $x[n]$ signal. As it has been mentioned before, the goal of this justbefore-test measurement is to evaluate the accuracy of the EMI measurement system close to the limit lines defined at the standards as it is the most critical amplitude for the verification. Therefore, it can be seen from Fig. 2, that the amplitudes of the tones are matching the limit amplitude for the quasi-peak detector defined at CISPR 32 standards for class B equipment.

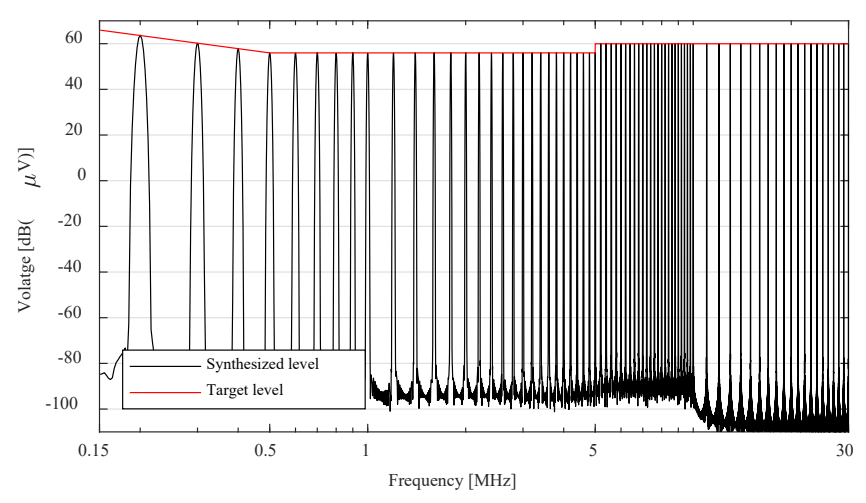

Fig. 2. Spectrum of the multi-tone waveform generated to evaluate the conducted emission test according to CISPR 32

b) Representative EUT interference to evaluate the accuracy of weighting detectors

The other waveform proposed to perform the just-beforetest verification for conducted emissions is a realistic excitation that can be commonly found at EUT. The purpose of this signal in comparison with the multi-tone waveform is to be capable of evaluating the different weighting detectors such us quasi-peak (QP) or average (AVG) defined in CISPR 16-1-1 [10]. This is very important as at the end the limits are defined with these detectors and not with the peak value in most of the common EMC standards. Moreover, with the representative interference, we are verifying that the test bench is performing accurately in front of interferences that are suitable to be expected in terms of amplitude and frequency shape.

Regarding the signal generated to excite the AWG, it is composed of a pulsed signal with a frequency repetition of 1 $\mathrm{kHz}$ and a duration of $10 \mu \mathrm{s}$, which will allow us to view differences with the different standard detectors. Moreover, the generated interference has also a ringing, which is a common phenomenon found at most of the measurements due to mismatch causes at the EUT design. This ringing is added with a Gaussian pulse waveform centered at $10 \mathrm{MHz}$ with a bandwidth of $5 \mathrm{MHz}$.

\section{2) Time-domain EMI measurement system}

To verify the different tests with the just-before-test measurement we propose to use novel measurement systems like TEMPS [11] and [12]. This measurement method is based on acquiring the time-domain signal employing generalpurpose oscilloscopes. Afterward, the spectral estimation is computed with a post-processing stage obtaining the equivalent resolution bandwidth and weighting detectors according to the CISPR 16-1-1 standard. 
In each acquisition, we are obtaining the full spectrum limited by the bandwidth of the oscilloscope and the acquisition configuration, so we are observing all the frequency range at each capture. In addition, compared with frequency sweep instrumentation, we have other advantages like the multichannel capability, which allow us to measure two or four lines simultaneously for conducted emissions, depending on whether mains is single or three phases. On the other hand, the cost of the instrumentation is lower compared with the use of instrumentation like VNA for just-before-test verification and the risk to damage it is minor as the input maximum voltage of the oscilloscope is of hundreds of volts compared to the few volts allowed by the VNA or a standard EMI receiver.

In Fig. 3, the basic schematic diagram of the time-domain systems is shown below.

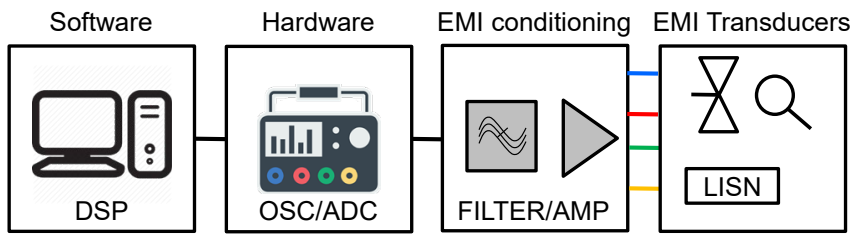

Fig. 3. The basic shematic diagram of the time-domain system

\section{B. Conducted immunity}

For the conducted immunity verification, the methodology will be based on placing the time-domain EMI measuring system instead of the EUT. Employing the previously described TEMPS system based on the use of an oscilloscope. The TEMPS system software will be run in a laptop capturing constantly the signal with a max-hold option. In this way, the measurement system is obtaining captures for instance for the frequency range between $150 \mathrm{kHz}$ and $80 \mathrm{MHz}$ continuously and computing the spectrum. Once the just-before-test system is ready, we run the immunity test increasing the frequency step from $1 \%$ to $20 \%$. This is more than sufficient for the verification purposes, because the failures commonly occur at broad ranges as it can be seen in the results section.

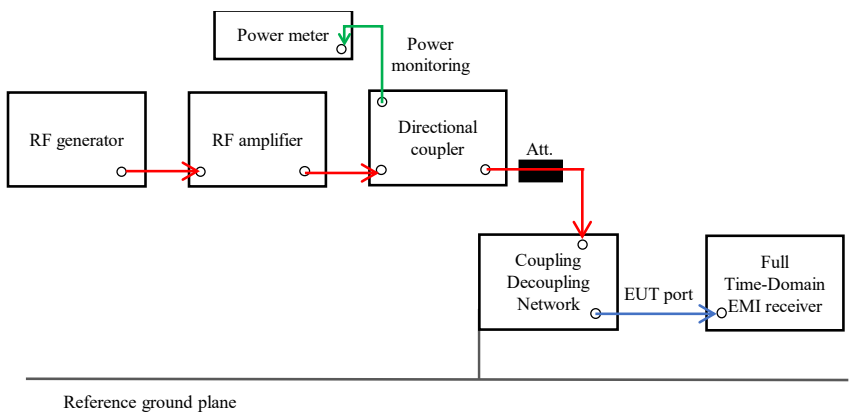

Fig. 4. Schematic of the just-before-test verification method for conducted immunity

The main advantage of this methodology is that we are evaluating the performance of the full chain. The generator, amplifier, paths, grounding and the coupling decoupling network. Therefore, if there is a failure in any of these items, the measurement of the voltage will not be in accordance with the calibrated test and failure can be reported by observing the deviation at each of the injected frequencies.

\section{RESULTS}

\section{A. Conducted emissions just-before-test verification}

With the aim to demonstrate the capabilities of the justbefore-test verification methodology, different scenarios have been created. A test set-up according to CISPR 32 is evaluated when everything is perfectly setup or different failures have been intentionally created. Therefore, the just-before-test methodology developed should allow us to identify malfunction of the test bench due to grounding or damage problems with the LISN, coupling planes, the receiver cables, and terminations.

To generate the reference signals described in the previous section a Keysight AWG model 81160A is used at the conducted emissions test instead of the EUT. The AWG is connected through a coaxial cable to the LISN of the test bench and the lines are measured employing the TEMPS system. The system is composed by the post-processing software and a Picoscope oscilloscope model 5444B used to capture the time-domain signal. The measurement time has been set to $100 \mathrm{~ms}$ with a sampling rate of $250 \mathrm{MSamples} / \mathrm{s}$.

Following, different measurements are presented when the test bench is working properly and when we create a delivered failure with the aim to observe if the just-before-test method is able to identify it. The first results shown in Fig. 5, are the results obtained for the multi-tone signal and the realistic interference when non-failure is introduced to the test set-up.

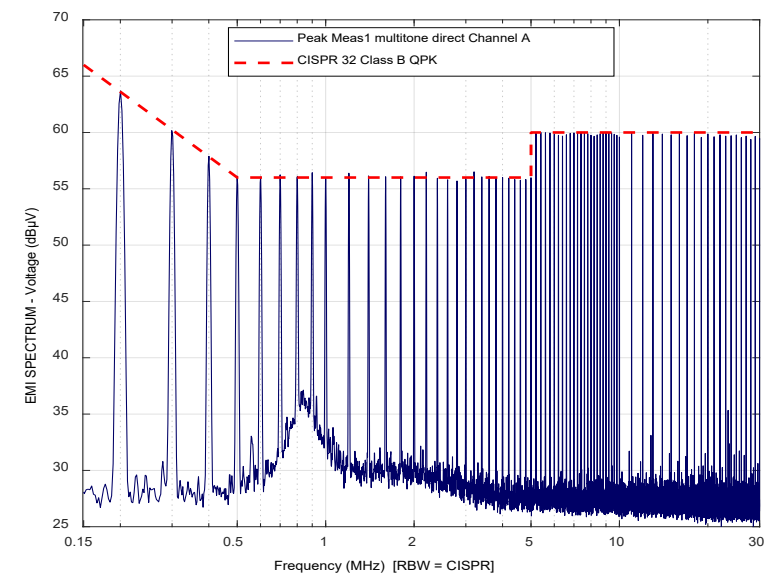

(a)

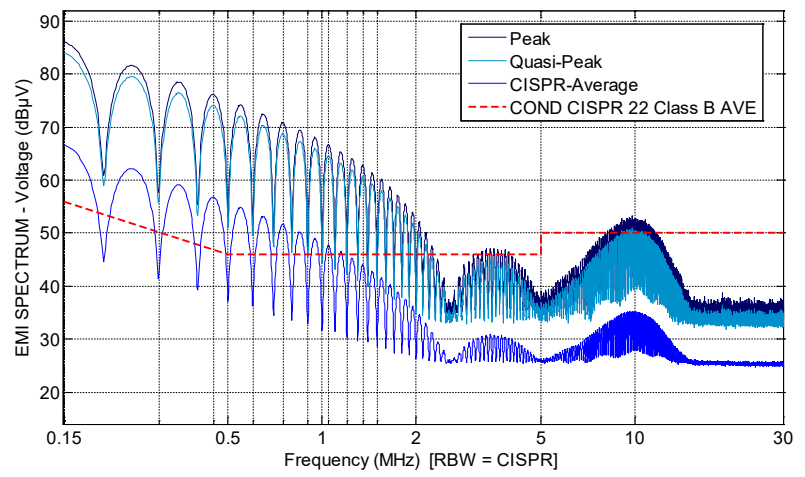

(b)

Fig. 5. Spectrum result when the multi-tone waveform is employed (a) and when the representative EUT interference is used (b)

From the results shown in Fig. 5 (a), we can see that the multi-tone signal is matching at each injected tone the level 
defined at CISPR 32 QP Class B limit line. Therefore, we can confirm that all the test bench is working properly when a nonfailure situation is present. However, from this measurement, we cannot determine anything from the mandatory weighting detectors defined at the standards. For this reason, we observe the realistic pulsed waveform generated by the AWG (Fig. 5(b)), on this occasion we compute the peak the QP and the AVG detectors. If we consider that the pulse repetition rate of the interference is $1 \mathrm{kHz}$, we can verify at the curved provided at Figure J.11 at CISPR 16-1-1 standard that the QP result and the AVG result are according to the illustrative curve. At this curve it is described that a $3 \mathrm{~dB}$ reduction for the QP measurement and a $20 \mathrm{~dB}$ reduction for the AVG one should appear in reference to the PEAK measurement, which is the reduction that can be observed in Fig. 5(b). Therefore, with these two measurements, we can ensure that the conducted emissions test bench for CISPR 32 standard is verified just before the test. Moreover, we have done these measurements with time-domain instrumentation and the total elapsed time to verify it is less than two minutes.

Following we produce deliverable failures and check the response of the just-before-test time-domain verification method. The first failure scenario that we are simulating is produced by a poor grounding connection between the LISN and the ground plane. In this occasion, we perform three different measurements with the multi-tone waveform. The first one is the reference and the LISN is properly connected to the ground plane via several metallic plates and screws, the second one is done by means of a metallic mesh and the last one is using a thin wire. The results of these measurements are illustrated in Fig. 6.

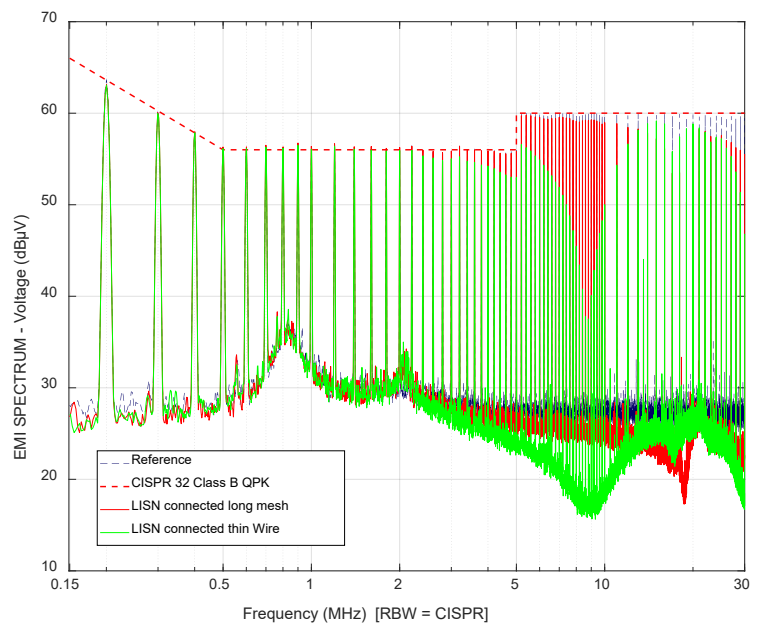

Fig. 6. Results for the different LISN grounding conditions. In blue the reference measurement, in red when the connection is done through a thin wire and in green when it is done by means of a mesh.

From the results, we can clearly observe the differences that are produced above $2 \mathrm{MHz}$ when the different grounding connections are used. The reference measurement is matching the limit line amplitudes, however, the long mesh and the thin wire are causing malfunction to the test bench. We can verify with the just-before-test measurement method that with the thin wire we have differences up to $20 \mathrm{~dB}$ at $8.5 \mathrm{MHz}$ and 5 $\mathrm{dB}$ at $30 \mathrm{MHz}$ with the mesh connection.

Hence, employing time-domain instrumentation in combination with the AWG, we are able to check if the entire test bench is working properly. The measurement time is less than $30 \mathrm{~s}$ and we are checking the instrumentation with the normative detectors, the paths, the coupling planes, the grounding, and the LISN.

\section{B. Conducted immunity just-before-test verification}

Similar to the conducted emissions, different cases have been evaluated with the just-before-test method developed for conducted immunity. We are able to identify failures at the $\mathrm{CDN}$ or injection clamp including damage, reverse placement or grounding failures. Additionally, we could evaluate the performance of the amplifier, RF generator, power meter, the path grounding and distance to the reference plane.

In this case, the TEMPS system is connected at the output of the EUT side of the CDN, emulating the EUT. The instrumentation employed to perform the just-before-test measurement is the same that has been used for the conducted emissions. As described before, the oscilloscope is placed instead of the EUT running TEMPS software, measuring continuously the RF with the max hold option. The oscilloscope employed is a Picoscope 5444B, which has a bandwidth of $200 \mathrm{MHz}$. Regarding the conducted immunity test, this is according to IEC 61000-4-6 standard. In order to perform the test, an R\&S SML03 signal generator is employed in combination with BONN BSA 0110-100 Power Amplifier, an AR PM2002 power Meter, and a Schlöder M2+M3 CDN. For the verification purposes, the frequency range is set between $150 \mathrm{kHz}$ and $80 \mathrm{MHz}$ with a frequency step of $20 \%$, as it is sufficient to evaluate the performance of the test bench. The dwell time at each frequency is set to $0.5 \mathrm{~s}$ and the target $\mathrm{RF}$ voltage is set to $3 \mathrm{~V}$. It is important to highlight that the time employed to perform the verification of the IEC 610004-6 is less than a minute, around 40 seconds.

The first scenario that we evaluate is when the test bench is working properly. The results can be observed in Fig. 7 in blue colour. The amplitude measured is $114 \mathrm{~dB} \mu \mathrm{V}$, which is the target amplitude divided by 6 as the load of the oscilloscope is set to $50 \mathrm{ohms}$. Otherwise, when we produce a failure scenario (see red line in Figure), where we place the $\mathrm{CDN}$ in reverse mode, the amplitude is not matching the theoretical value and the frequency response is not flat. In fact, in some frequencies, we are receiving $28 \mathrm{~dB}$ less than the expected value. Therefore, with the just-before-test verification method, we can identify this type of defect that would have been missed if we had used a VNA to check the paths or we had just been monitoring the forward and reverse power at the power meter.

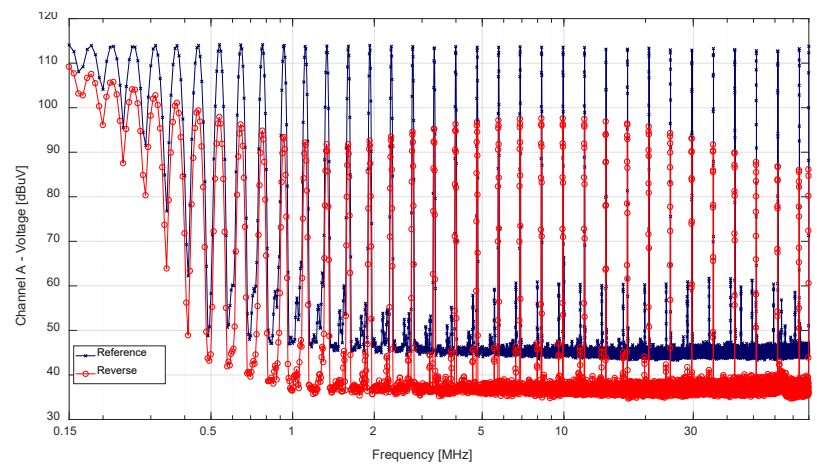

Fig. 7. Results of the just-before-test verification method when the CDN is placed in reverse position (red colour) compared with the reference in blue.

The other scenario that we have simulated is the case in which the CDN is not properly connected to the reference ground plane. In this occasion, we have connected the CDN through 
a thin wire and we have disconnected the CDN from the ground plane using an isolation material of $5 \mathrm{~mm}$. The results of this scenario are shown in Fig. 8, where the blue trace is the reference measurement, the green one is the $\mathrm{CDN}$ connected using the thin wire and the red trace is the result of the CDN disconnected from the ground plane.

From the results, it is noticeable that the inadequate connection of the CDN produces a strong impact on the test. Having a higher influence at the lowest frequency range, where differences up to $34 \mathrm{~dB}$ can be found compared with the reference scenario. Therefore, it is crucial to ensure the correct grounding with all the available CDN surface. Otherwise, the just-before test methodology has been able to detect this failure on the connection.

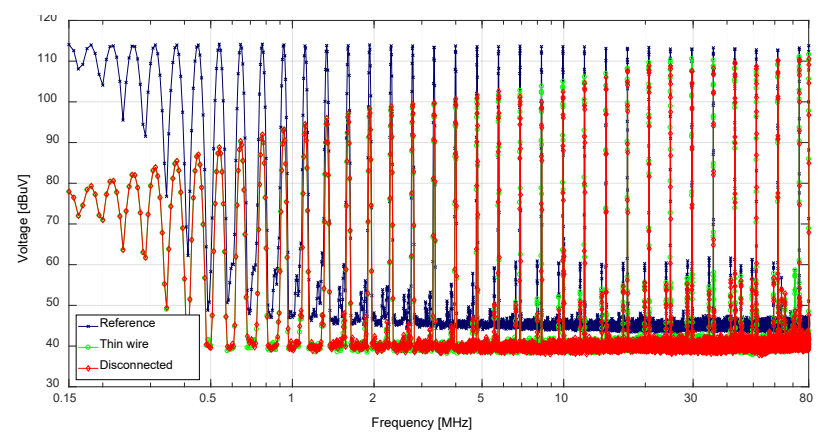

Fig. 8. Results of the just-before-test verification method when the CDN is connected to the ground plane in a poor manner.

\section{CONCLUSIONS}

In this paper, two different just-before-test methodologies have been developed to verify the proper functionality of the conducted emissions and immunity tests. Both of the methodologies are based on the use of time-domain measurement systems employing oscilloscopes at the acquisition stage, which allows us to verify the test in less than a minute for conducted immunity and in two minutes for conducted emissions. As it has been mentioned at the introduction section, the speed and ease of setup are critical for the daily use at the EMC test laboratories and both are accomplished with the proposed methodologies. Moreover, novel advantages are introduced by the use of controlled waveform generated by arbitrary waveform generators and the use of oscilloscopes for emissions and immunity tests. Regarding the arbitrary waveform, it allows us to evaluate the full chain of the emissions test including the weighting detectors and ensuring the accuracy of the measurement around the limit line defined at the standards. On the other hand, the employment of the oscilloscope at the immunity test allows us to evaluate the performance of the path, CDN and novelty the performance of the generator and the power amplifier.

At the results section, several experiments simulating failures have been evaluated with the goal to verify if the justbefore-test method were able to identify the defective scenarios. In all the occasions the developed methodologies were functioning accurately and defects like grounding or reverse CDN connection have been detected. Therefore, the rapid and cheap methodologies have been validated in the experimental section and should be suitable to be implement by EMC test laboratories as a new tool to be in compliance with the requirements of test verification. A discussion might be if this type of verification methods should be included at the EMC standards, in order that all the test laboratories can apply them. Increasing the quality of the test laboratory and clearly identifying major failures like path or instrumentation damage or measurement accuracy problems just-before-test.

\section{ACKNOWLEDGMENT}

This work was supported by the project 15RPT01 RFMicrowave. This project has received funding from the EMPIR programme co-financed by the Participating States and from the European Union's Horizon 2020 research and innovation programme.

\section{REFERENCES}

[1] ISO IEC, 17025 ed3.0: General requirements for the competence of testing and calibration laboratories. ISO/IEC, 2017.

[2] E. Tas and F. Pythoud, "Design, Implementation, and Evaluation of Proficiency Testing in EMC Conducted Immunity," in IEEE Transactions on Electromagnetic Compatibility, vol. 59, no. 5, pp. 1433-1440, Oct. 2017.

[3] E. Tas et al., "Proficiency testing for conducted immunity with a new round robin test device," 2016 International Symposium on Electromagnetic Compatibility - EMC EUROPE, Wroclaw, 2016, pp. 274-279.

[4] 15RPT01 RFMicrowave. Improved microwave measurement capabilities will boost quality and confidence in advanced technologies. http://www.rfmw.cmi.cz/

[5] O. Şen and S. Çakir, "Improved Just-Before-Test Verification Methods with VNA for Conducted EMC Tests," 2018 International Symposium on Electromagnetic Compatibility (EMC EUROPE), Amsterdam, 2018, pp. 488-493.

[6] K. Sieczkarek and A. Mackowiak, "European intercomparison exercises - conducted, radiated emission and field verification for immunity tests," 2005 International Symposium on Electromagnetic Compatibility, 2005. EMC 2005., Chicago, IL, 2005, pp. 118-123 Vol.

[7] IEC, 61000-4-6 ed4.0: Electromagnetic compatibility (EMC) - Part 46: Testing and measurement techniques - Immunity to conducted disturbances, induced by radio-frequency fields. IEC, 2013

[8] M. A. Azpúrua, M. Pous, J. A. Oliva, B. Pinter, M. Hudlička and F. Silva, "Waveform Approach for Assessing Conformity of CISPR 161-1 Measuring Receivers," in IEEE Transactions on Instrumentation and Measurement, vol. 67, no. 5, pp. 1187-1198, May 2018.

[9] M. Friese, "Multitone signals with low crest factor," IEEE Trans.Commun., vol. 45, no. 10, pp. 1338-1344, Oct. 1997.

[10] IEC CISPR, 16-1-1 ed4.0: Specification for radio disturbance and immunity measuring apparatus and methods - Part 1-1: Radio disturbance and immunity measuring apparatus - Measuring apparatus. IEC, 2015.

[11] M. A. Azpúrua, M. Pous, M. Femandez and F. Silva, "Dynamic Performance Evaluation of Full Time Domain EMI Measurement Systems," 2018 International Symposium on Electromagnetic Compatibility (EMC EUROPE), Amsterdam, 2018, pp. 561-566.

[12] M. A. Azpúrua, J. A. Oliva, M. Pous and F. Silva, "Fast and automated verification of multi-channel full time-domain EMI measurement systems," 2017 IEEE International Instrumentation and Measurement Technology Conference (I2MTC), Turin, 2017, pp. 1-6. 